\title{
Applications of (Neutro/Anti)sophications to Semihypergroups
}

\author{
A. Rezaei $\left(\mathbb{D},{ }^{1}\right.$ F. Smarandache $\mathbb{D}^{2},{ }^{2}$ and S. Mirvakili $\mathbb{D}^{1}$ \\ ${ }^{1}$ Department of Mathematics, Payame Noor University, P.O. Box 19395-3697, Tehran, Iran \\ ${ }^{2}$ Department of Mathematics and Science, University of New Mexico, Gallup, New Mexico 87301, USA
}

Correspondence should be addressed to A. Rezaei; rezaei@pnu.ac.ir

Received 17 December 2020; Revised 18 February 2021; Accepted 25 February 2021; Published 30 March 2021

Academic Editor: Parimala Mani

Copyright (c) 2021 A. Rezaei et al. This is an open access article distributed under the Creative Commons Attribution License, which permits unrestricted use, distribution, and reproduction in any medium, provided the original work is properly cited.

In this paper, we extend the notion of semi-hypergroups (resp. hypergroups) to neutro-semihypergroups (resp. neutrohypergroups). We investigate the property of anti-semihypergroups (resp. anti-hypergroups). We also give a new alternative of neutro-hyperoperations (resp. anti-hyperoperations), neutro-hyperoperation-sophications (resp. anti-hypersophications). Moreover, we show that these new concepts are different from classical concepts by several examples.

\section{Introduction}

A hypergroup, as a generalization of the notion of a group, was introduced by F. Marty [1] in 1934. The first book in hypergroup theory was published by Corsini [2]. Nowadays, hypergroups have found applications to many subjects of pure and applied mathematics, for example, in geometry, topology, cryptography and coding theory, graphs and hypergraphs, probability theory, binary relations, theory of fuzzy and rough sets and automata theory, physics, and also in biological inheritance [3-7]. The first book in semihypergroup theory was published by Davvaz in 2016 (see [8]). In recent years, several other valuable books in hyperstructures have been written by Davvaz et al. $[6,9,10]$.

M. Al-Tahan et al. introduced the Corsini hypergroup and studied its properties as a special hypergroup that was defined by Corsini. They investigated a necessary and sufficient condition for the productional hypergroup to be a Corsini hypergroup, and they characterized all Corsini hypergroups of orders 2 and 3 up to isomorphism [3]. Semihypergroup, hypergroup, and fuzzy hypergroup of order 2 are enumerated in $[7,11,12]$. S. Hoskova-Mayerova et al. used the fuzzy multisets to introduce the concept of fuzzy multi-hypergroups as a generalization of fuzzy hypergroups, defined the different operations on fuzzy multi-hypergroups, and extended the fuzzy hypergroups to fuzzy multihypergroups [13].
In 2019 and 2020, within the field of neutrosophy, Smarandache [14-16] generalized the classical algebraic structures to neutroalgebraic structures (or neutroalgebras) (whose operations and axioms are partially true, partially indeterminate, and partially false) as extensions of partial algebra and to antialgebraic structures (or antialgebras) (whose operations and axioms are totally false). Furthermore, he extended any classical structure, no matter what field of knowledge, to a neutrostructure and an antistructure. These are new fields of research within neutrosophy. Smarandache in [16] revisited the notions of neutroalgebras and antialgebras, where he studied partial algebras, universal algebras, effect algebras, and Boole's partial algebras and showed that neutroalgebras are the generalization of partial algebras. Also, with respect to the classical hypergraph (that contains hyperedges), Smarandache added the supervertices (a group of vertices put together to form a supervertex), in order to form a super-hypergraph. Then, he extended the superhypergraph to $n$-super-hypergraph, by extending the power set $P(V)$ to $P^{n}(V)$ that is the $n$-power set of the set $V$ (the $n$ super-hypergraph, through its $n$-super-hypergraph-vertices and $n$-superhypergraph-edges that belong to $P^{n}(V)$, can be the best (so far) to model our complex and sophisticated reality). Furthermore, he extended the classical hyperalgebra to $n$-ary hyperalgebra and its alternatives $n$-ary neutrohyperalgebra and $n$-ary anti-hyperalgebra [17]. The notion of neutrogroup was defined and studied by Agboola in [18] 
Recently, M. Al-Tahan et al. studied neutro-ordered algebra and some related terms such as neutro-ordered subalgebra and neutro-ordered homomorphism in [19].

In this paper, the concept of neutro-semihypergroup and anti-semihypergroup is formally presented. And, new alternatives are introduced, such as neutro-hyperoperations (resp. anti-hyperoperations), neutro-hyperaxioms, and antihyperaxioms. We show that these definitions are different from classical definitions by presenting several examples. Also, we enumerate neutro-hypergroup and anti-hypergroup of order 2 (see Table 1) and obtain some known results (see Table 2).

\section{Preliminaries}

In this section, we recall some basic notions and results regarding hyperstructures.

Definition 1 (see $[2,8])$. A hypergroupoid $(H, \circ)$ is a nonempty set $H$ together with a map $\circ: H \times H \longrightarrow P^{*}(H)$ called (binary) hyperoperation, where $P^{*}(H)$ denotes the set of all nonempty subsets of $H$. The hyperstructure $(H, \circ)$ is called a hypergroupoid, and the image of the pair $(x, y)$ is denoted by $x \circ y$.

If $A$ and $B$ are nonempty subsets of $H$ and $x \in H$, then by $A \circ B, A \circ x$, and $x \circ B$ we mean $A \circ B=\cup_{a \in A, b \in B} a \circ b$, $A \circ x=A \circ\{x\}$, and $x \circ B=\{x\} \circ B$.

Definition 2 (see $[2,8]$ ). A hypergroupoid $(H, \circ)$ is called a semi-hypergroup if it satisfies the following:

(A) $(\forall a, b, c \in H)(a \circ(b \circ c)=(a \circ b) \circ c)$ (associativity).

Definition 3 (see $[2,8]$ ). A hypergroupoid $(H, \circ)$ is called a quasi-hypergroup if reproduction axiom is valid. This means that, for all $a$ of $H$, we have

$$
\begin{aligned}
& \text { (R) } \quad(\forall a \in H)(H \circ a=a \circ H=H) \quad \text { (i.e. } \quad(\forall a, b \in H) \\
& (\exists c, d \in H) \text { s.t. } b \in c \circ a, b \in a \circ d) .
\end{aligned}
$$

Definition 4 (see $[2,8]$ ). A hypergroupoid $(H, \circ)$ which is both a semi-hypergroup and a quasi-hypergroup is called a hypergroup.

\section{Example 1 (see $[2,8]$ )}

(i) Let $H$ be a nonempty set, and for all $x, y \in H$, we define $x \circ y=H$. Then, $(H, \circ)$ is a hypergroup, called the total hypergroup.

(ii) Let $G$ be a group and $H$ a normal subgroup of $G$, and for all $x, y \in G$, we define $x \circ y=x y H$. Then, $(G, \circ)$ is a hypergroup.

Definition 5 (see $[2,12])$. Let $(H, \circ)$ be a hypergroupoid. The commutative law on $(H, \circ)$ is defined as follows:

(C) $(\forall a, b \in H)(a \circ b=b \circ a)$.

$(H, \circ)$ is called a commutative hypergroupoid.
TABLE 1: Classification of the hypergroupoids of order 2.

\begin{tabular}{ccccc}
\hline & & A & NA & AA \\
\hline \multirow{4}{*}{ C } & R & 6 & 4 & - \\
& NR & - & - & - \\
& AR & - & - & - \\
& Etc. & 3 & 2 & - \\
NC & R & - & - & - \\
& NR & - & - & - \\
& AR & - & - & - \\
& Etc. & - & - & - \\
AC & R & 2 & 8 & - \\
& NR & - & - & - \\
& AR & - & - & 4 \\
\hline
\end{tabular}

TABle 2: Classification of the semi-hypergroups of order 2.

\begin{tabular}{lccc}
\hline & Com & Noncom & $N$ \\
\hline Semigroup & 3 & 2 & 5 \\
Group & 1 & - & 1 \\
Semi-hypergroup & 9 & 8 & 17 \\
Hypergroup & 6 & 2 & 8 \\
\hline
\end{tabular}

Example 2 (see [13]). Let $\mathbb{Z}$ be the set of integers, and define ${ }^{\circ}$ on $\mathbb{Z}$ as follows. For all $x, y \in \mathbb{Z}$,

$$
x \circ{ }_{1} y=\left\{\begin{array}{ll}
2 \mathbb{Z}, & \text { if } x, y \text { have same partiy, } \\
2 \mathbb{Z}+1, & \text { otherwise. }
\end{array}\right\} .
$$

Then, $\left(\mathbb{Z}, \circ_{1}\right)$ is a commutative hypergroup.

\section{On Neutro-hypergroups and Anti- hypergroups}

F. Smarandache generalized the classical algebraic structures to the neutroalgebraic structures and antialgebraic structures. Neutro-sophication of an item $C$ (that may be a concept, a space, an idea, a hyperoperation, an axiom, a theorem, a theory, an algebra, etc.) means to split $C$ into three parts (two parts opposite to each other, and another part which is the neutral/indeterminacy between the opposites), as pertinent to neutrosophy $((\langle A\rangle,\langle$ neut $A\rangle$, $\langle\operatorname{anti} A\rangle)$, or with other notation $(T, I, F))$, meaning cases where $C$ is partially true $(T)$, partially indeterminate $(I)$, and partially false $(F)$, while antisophication of $C$ means to totally deny $C$ (meaning that $C$ is made false on its whole domain) (see $[14,15,17,20])$.

Neutrosophication of an axiom on a given set $X$ means to split the set $X$ into three regions such that, on one region, the axiom is true (we say the degree of truth $T$ of the axiom), on another region, the axiom is indeterminate (we say the degree of indeterminacy $I$ of the axiom), and on the third region, the axiom is false (we say the degree of falsehood $F$ of the axiom), such that the union of the regions covers the whole set, while the regions may or may not be disjoint, where $(T, I, F)$ is different from $(1,0,0)$ and from $(0,0,1)$.

Antisophication of an axiom on a given set $X$ means to have the axiom false on the whole set $X$ (we say total degree of falsehood $F$ of the axiom) or $(0,0,1)$. 
Neutrosophication of a hyperoperation defined on a given set $X$ means to split the set $X$ into three regions such that, on one region, the hyperoperation is well-defined (or inner-defined) (we say the degree of truth $T$ of the hyperoperation), on another region, the hyperoperation is indeterminate (we say the degree of indeterminacy $I$ of the hyperoperation), and on the third region, the hyperoperation is outer-defined (we say the degree of falsehood $F$ of the hyperoperation), such that the union of the regions covers the whole set, while the regions may or may not be disjoint, where $(T, I, F)$ is different from $(1,0,0)$ and from $(0,0,1)$.

Antisophication of a hyperoperation on a given set $X$ means to have the hyperoperation outer-defined on the whole set $X$ (we say total degree of falsehood $F$ of the axiom) or $(0,0,1)$.

In this section, we will define the neutro-hypergroups and anti-hypergroups.

Definition 6. A neutro-hyperoperation is a map $\circ: H \times H \longrightarrow P(U)$, where $U$ is a universe of discourse that contains $H$ that satisfies the below neutrosophication process.

The neutrosophication (degree of well-defined, degree of indeterminacy, and degree of outer-defined) of the hyperoperation is the following neutrohyperoperation $(\mathrm{NH})$ :

(NR) $\quad(\exists x, y \in H)\left(x \circ y \in P^{*}(H)\right)$ and $(\exists x, y \in$ $H)\left(x \circ y\right.$ is an indeterminate subset, or $\left.x \circ y \notin P^{*}(H)\right)$.

The neutrosophication (degree of truth, degree of indeterminacy, and degree of falsehood) of the hypergroup axiom of associativity is the following neutroassociativity (NA):

(NA) $(\exists a, b, c \in H)(a \circ(b \circ c)=(a \circ b) \circ c)$ and $(\exists d, e$, $f \in H)(d \circ(e \circ f) \neq(d \circ e) \circ f$ or $d \circ(e \circ f)=$ indeterminate, or $(d \circ e) \circ f=$ indeterminate $)$.

Neutroreproduction axiom (NR):

(NR) $(\exists a \in H)(H \circ a=a \circ H=H)$ and $(\exists b \in H)$ ( $H \circ b, b \circ H$, and $H$ are not all three equal, or some of them are indeterminate).

Also, we define the neutrocommutativity (NC) on $(H, \circ)$ as follows:

(NC) $(\exists a, b \in H)(a \circ b=b \circ a) \quad$ and $\quad(\exists c, d \in H)$ $(c \circ d \neq d \circ c$, or $c \circ d=$ indeterminate, or $d \circ c=$ indeterminate).

Now, we define a neutro-hyperalgebraic system $S=\langle H, F, A\rangle$, where $H$ is a set or neutrosophic set, $F$ is a set of the hyperoperations, and $A$ is the set of hyperaxioms, such that there exists at least one neutro-hyperoperation or at least one neutro-hyperaxiom and no anti-hyperoperation and no anti-hyperaxiom.

Definition 7. The anti-hypersophication (totally outerdefined) of the hyperoperation defines anti-hyperoperation $(\mathrm{AH}):(\mathrm{AH})(\forall x, y \in H)\left(x \circ y \notin P^{*}(H)\right)$.

The anti-hypersophication (totally false) of the hypergroup is as follows:
(AA) $(\forall x, y, z \in H)(x \circ(y \circ z) \neq(x \circ y) \circ z)$

(antiassociativity)

(AR) $(\forall a \in H)(H \circ a, a \circ H$, and $H$ are not equal) (antireproduction axiom)

Also, we define the anticommutativity $(\mathrm{AC})$ on $(H, \circ)$ as follows:

(AC) $(\forall a, b \in H$ with $a \neq b)(a \circ b \neq b \circ a)$.

Definition 8. A neutro-semihypergroup is an alternative of semi-hypergroup that has at least $(\mathrm{NH})$ or $(\mathrm{NA})$, which does not have (AA).

Example 3

(i) Let $H=\{a, b, c\}$ and $U=\{a, b, c, d\}$ be a universe of discourse that contains $H$. Define the neutrohyperoperation ${ }_{2}$ on $H$ with Cayley's table.

\begin{tabular}{c|ccc}
$\circ_{2}$ & $a$ & $b$ & $c$ \\
\hline$a$ & $a$ & $a$ & $a$ \\
$b$ & $b$ & $\{a, b\}$ & $\{a, b, d\}$ \\
$c$ & $c$ & $?$ & $H$
\end{tabular}

Then, $\left(H, \circ_{2}\right)$ is a neutro-semihypergroup. Since $a \circ_{2} b \in P^{*}(H), \quad b \circ_{2} c=\{a, b, d\} \notin P^{*}(H), \quad$ and $c \circ_{2} b=$ indeterminate, so $(\mathrm{NH})$ holds.

(ii) Let $H=\{a, b, c\}$. Define the hyperoperation ${ }_{3}$ on $H$ with Cayley's table.

\begin{tabular}{c|ccc}
$\circ_{3}$ & $a$ & $b$ & $c$ \\
\hline$a$ & $a$ & $a$ & $a$ \\
$b$ & $b$ & $\{a, b\}$ & $\{a, b\}$ \\
$c$ & $c$ & $\{b, c\}$ & $H$
\end{tabular}

Then, $\left(H, \circ_{3}\right)$ is a neutro-semihypergroup. (NA) is valid, since $\left(b \circ_{3} c\right) \circ_{3} a=\{a, b\} \circ_{3} a=\left(a \circ_{3} a\right) \cup\left(b \circ \circ_{3} a\right)=\{a\} \cup$ $\{b\}=\{a, b\}$ and $b \circ_{3}\left(c \circ_{3} a\right)=b \circ_{3}\{c\}=b \circ_{3} c=\{a, b\}$.

Hence, $\left(b \circ_{3} c\right) \circ_{3} a=b \circ_{3}\left(c \circ_{3} a\right)$. Also, $\left\{b \circ_{3} a\right\} \circ_{3}$ $c=\{b\} \circ_{3} c=b \circ_{3} c=\{a, b\}$ and $b \circ_{3}\left(a \circ_{3} c\right)=b \circ_{3}\{a\}=$ $b \circ_{3} a=\{b\}$, so $\left(b \circ_{3} a\right) \circ_{3} c \neq b \circ_{3}\left(a \circ_{3} c\right)$.

Definition 9. A neutrocommutative semi-hypergroup is a semi-hypergroup that satisfies (NC).

Example 4. Let $H=\{a, b, c\}$. Define the hyperoperation ${ }^{\circ}$ on $H$ with Cayley's table.

\begin{tabular}{c|ccc}
$\circ_{4}$ & $a$ & $b$ & $c$ \\
\hline$a$ & $\{a, c\}$ & $a$ & $a$ \\
$b$ & $a$ & $b$ & $c$ \\
$c$ & $a$ & $\{b, c\}$ & $\{b, c\}$
\end{tabular}

Then, $\left(H,{ }_{4}\right)$ is a semi-hypergroup, but not a hypergroup, since $a^{\circ}{ }_{4} H=H^{\circ}{ }_{4} a=\{a, c\} \neq H$. (NC) is valid, since $a \circ_{4} b=\{a\}=b \circ_{4} a$ and $c \circ_{4} b=\{b, c\} \neq b \circ_{4} c=\{c\}$. 
Definition 10. A neutrocommutative hypergroup is a hypergroup that satisfies (NC).

Example 5. Let $H=\{a, b, c, d, e, f\}$. Define the operation ${ }_{5}$ on $H$ with Cayley's table.

\begin{tabular}{c|llllll}
$\circ_{5}$ & $a$ & $b$ & $c$ & $d$ & $e$ & $f$ \\
\hline$e$ & $e$ & $a$ & $b$ & $c$ & $d$ & $f$ \\
$a$ & $a$ & $b$ & $e$ & $d$ & $f$ & $c$ \\
$b$ & $b$ & $e$ & $a$ & $f$ & $c$ & $d$ \\
$c$ & $c$ & $f$ & $d$ & $e$ & $b$ & $a$ \\
$d$ & $d$ & $c$ & $f$ & $a$ & $e$ & $b$ \\
$f$ & $f$ & $d$ & $c$ & $b$ & $a$ & $e$
\end{tabular}

Then, $\left(H, o_{5}, e\right)$ is a group and so is a natural hypergroup. Also, it is a neutrocommutative hypergroup, since $a \circ_{5} b=e=b \circ_{5} a$ and $a \circ_{5} c=d \neq c \circ_{5} a=f$.

Definition 11. A neutrohypergroup is an alternative of hypergroup that has at least $(\mathrm{NH})$ or $(\mathrm{NA})$ or $(\mathrm{NR})$, which does not have (AA) and (AR).

Example 6. Let $H=\{a, b, c\}$. Define the hyperoperation ${ }_{6} 6$ on $H$ with Cayley's table.

$$
\begin{array}{c|ccc}
\circ_{6} & a & b & c \\
\hline a & a & b & c \\
b & b & b & b \\
c & c & c & a
\end{array}
$$

Then, $\left(H, \circ_{6}\right)$ is a neutrohypergroup. The hyperoperation ${ }_{6} 6$ is associative. (NR) is valid, since $a \circ_{6} H=\left(a \circ_{6} a\right) \cup\left(a \circ_{6} b\right) \cup\left(a \circ_{6} c\right)=H=\left(a \circ_{6} a\right) \cup\left(b \circ{ }_{6} a\right)$ $\cup\left(c \circ_{6} a\right)=H \circ_{6} a, \quad b \circ_{6} H=\left(b \circ_{6} a\right) \cup\left(b \circ{ }_{6} b\right) \cup\left(b \circ_{6} c\right)=$ $\{b\} \neq H \neq\{c, b\}=\left(a \circ{ }_{6} b\right) \cup\left(b \circ{ }_{6} b\right) \cup\left(c \circ{ }_{6} b\right)=H \circ_{6} b$, and $c \circ_{6} H=\left(c \circ_{6} a\right) \cup\left(c \circ_{6} b\right) \cup\left(c \circ_{6} c\right)=\{a, c\} \neq H$, but $H \circ_{6} c=$ $\left(a \circ{ }_{6} c\right) \cup\left(b \circ{ }_{6} c\right) \cup\left(c \circ{ }_{6} c\right)=\{a, b, c\}=H$.

Note that every neutro-semihypergroup, neutrohypergroup, neutrocommutative semi-hypergroup, and neutrocommutative hypergroup are neutro-hyperalgebraic systems.

Definition 12. An anti-semihypergroup is an alternative of semi-hypergroup that has at least $(\mathrm{AH})$ or $(\mathrm{AA})$.

\section{Example 7}

(i) Let $\mathbb{N}$ be the set of natural numbers except 0 . Define hyperoperation $\circ_{7}$ on $\mathbb{N}$ by $x \circ_{7} y=\left\{\left(x^{2} / x^{2}+1\right), y\right\}$. Then, $\left(\mathbb{N}, \circ_{7}\right)$ is an anti-semihypergroup. (AH) is valid, since, for all $x, y \in \mathbb{N}, x \circ_{7} y \notin P^{*}(\mathbb{N})$. Thus, $(\mathrm{AH})$ holds.

(ii) Let $H=\{a, b\}$. Define the hyperoperation ${ }_{8}$ on $H$ with Cayley's table.

$$
\begin{array}{l|ll}
\circ_{8} & a & b \\
\hline a & b & a \\
b & b & a
\end{array}
$$

Then, $\left(H, \circ_{8}\right)$ is an anti-semihypergroup. (AA) is valid, since, for all $x, y, z \in H, \quad x \circ_{8}\left(y \circ_{8} z\right) \neq$ $\left(x \circ{ }_{8} y\right) \circ_{8} z$.

(iii) Let $H=\{a, b\}$. Define the hyperoperation $\circ_{9}$ on $H$ with Cayley's table.

\begin{tabular}{l|ll}
$\circ_{9}$ & $a$ & $b$ \\
\hline$a$ & $b$ & $H$ \\
$b$ & $a$ & $a$
\end{tabular}

Then, $\left(H, \circ_{9}\right)$ is an anticommutative semi-hypergroup. (AC) is valid, since $a \circ_{9} b=H \neq b \circ_{9} a=\{a\}$.

Definition 13. An anti-hypergroup is an antisemihypergroup, or it satisfies (AR).

\section{Example 8}

(i) Let $\mathbb{R}$ be the set of real numbers. Define hyperoperation ${ }^{\circ}{ }_{10}$ on $\mathbb{R}$ by $x \circ_{10} y=\left\{x^{2}+1, x^{2}-1\right\}$. Then, $\left(\mathbb{R},{ }_{10}\right)$ is an anti-semihypergroup, since, for all $x, y, z \in \mathbb{R}, \quad x \circ_{10}\left(y \circ_{10} z\right) \neq\left(x \circ_{10} y\right) \circ_{10} z$. Because $\quad x \circ_{10}\left(y \circ{ }_{10} z\right)=x \circ_{10}\left\{y^{2}+1, y^{2}-1\right\}=$ $\left\{x \circ_{10}\left(y^{2}+1\right), x \circ_{10}\left(y^{2}-1\right)\right\}=\left\{x^{2}+1, x^{2}-1\right\}$, but $\left(x \circ_{10} y\right) \circ_{10} z=\left\{x^{2}+1, x^{2}-1\right\} \circ_{10} z=\left(\left(x^{2}+1\right)\right.$ $\left.{ }^{\circ}{ }_{10} z\right) \cup\left(\left(x^{2}-1\right) \circ_{10} z\right)=\left\{\left(x^{2}+1\right)^{2}+1,\left(x^{2}-1\right)^{2}\right.$ $+1\}$. Hence, (AA) is valid.

(ii) Let $H=\{a, b, c\}$. Define the hyperoperation ${ }^{\circ}{ }_{11}$ on $H$ with Cayley's table.

\begin{tabular}{c|ccc}
$\circ_{11}$ & $a$ & $b$ & $c$ \\
\hline$a$ & $a$ & $a$ & $b$ \\
$b$ & $a$ & $a$ & $a$ \\
$c$ & $c$ & $c$ & $c$
\end{tabular}

Then, $\left(H, \circ_{11}\right)$ is an anti-semihypergroup. The hyperoperation ${ }^{\circ}{ }_{11}$ is associative. Also, (AR) holds, since $a \circ{ }_{11} H=\left(a \circ{ }_{11} a\right) \cup\left(a \circ{ }_{11} b\right) \cup\left(a \circ{ }_{11} c\right)=\{c\} \neq$ $H \neq\{b, c\}=\left(a \circ{ }_{11} a\right) \cup\left(b \circ{ }_{11} a\right) \cup\left(c \circ{ }_{11} a\right)=H \circ_{11} a$, $b \circ_{11} H=\left(b \circ{ }_{11} a\right) \cup\left(b \circ{ }_{11} b\right) \cup\left(b \circ{ }_{11} c\right)=\{b\} \neq H \neq$ $\{b, c\}=\left(a \circ{ }_{11} b\right) \cup\left(b \circ \circ_{11} b\right) \cup\left(c \circ_{11} b\right)=H \circ_{11} b$, and $c \circ{ }_{11} H=\left(c \circ{ }_{11} a\right) \cup\left(c \circ{ }_{11} b\right) \cup \quad\left(c \circ{ }_{11} c\right)=\{c\} \neq H \neq$ $\{b, c\}=\left(a \circ{ }_{11} c\right) \cup\left(b \circ{ }_{11} c\right) \cup\left(c \circ{ }_{11} c\right)=H \circ{ }_{11} c$.

(iii) Let $\mathbb{R}$ be the set of real numbers. Define hyperoperation $\circ_{12}$ on $\mathbb{R}$ by $x \circ_{12} y=\{x, 1\}$. Then, $\left(\mathbb{R},{ }_{12}\right)$ is an anti-semihypergroup. The hyperoperation ${ }^{\circ}{ }_{12}$ is associative, since, for all $x, y, z \in \mathbb{R}$, we have $x \circ_{12}\left(y \circ_{12} z\right)=x \circ_{12}\{y, 1\}=\left(x \circ_{12} y\right) \cup$ $\left(x \circ{ }_{12} 1\right)=\{x, 1\} \cup\{x, 1\}=\{x, 1\}$ and $\left(x \circ_{12} y\right) \circ{ }_{12} z=$ $\{x, 1\} \circ_{12} z=\left(x \circ \circ_{12} z\right) \cup\left(1 \circ{ }_{12} z\right)=\{x, 1\} \cup\{1,1\}=$ $\{x, 1\}$, so $x \circ_{12}\left(y \circ_{12} z\right)=\left(x \circ_{12} y\right) \circ{ }_{12} z$. However, for $a \in \mathbb{R}$, we have $a \circ{ }_{12} \mathbb{R}=\cup_{x \in \mathbb{R}} a{ }_{12} x=U_{x \in \mathbb{R}}$ 


$$
\begin{aligned}
& \{a, 1\}=\{a, 1\} \neq \mathbb{R} \text { and } R \circ{ }_{12} a=\quad \cup_{x \in \mathbb{R}} x \circ{ }_{12} a= \\
& \cup_{x \in R}\{x, 1\}=\mathbb{R} \text {. Thus, } a \circ{ }_{12} \mathbb{R} \neq \mathbb{R} \circ{ }_{12} a .
\end{aligned}
$$

Definition 14. An anticommutative semi-hypergroup is a semi-hypergroup that satisfies (AC).

\section{Example 9}

(i) Let $H=\{a, b\}$. Define the hyperoperation ${ }^{\circ}{ }_{13}$ on $H$ with Cayley's table.

\begin{tabular}{l|ll}
$\circ_{13}$ & $a$ & $b$ \\
\hline$a$ & $a$ & $a$ \\
$b$ & $H$ & $b$
\end{tabular}

Then, $\left(H,{ }_{13}\right)$ is a semi-hypergroup and $(\mathrm{AC})$ is valid, since $a \circ{ }_{13} b=\{a\} \neq b \circ{ }_{13} a=H$. Thus, $\left(H,{ }_{13}\right)$ is an anticommutative semi-hypergroup.

(ii) Let $H=\{a, b\}$. Define the hyperoperation ${ }^{\circ}{ }_{14}$ on $H$ with Cayley's table.

\begin{tabular}{l|ll}
$\circ_{14}$ & $a$ & $b$ \\
\hline$a$ & $b$ & $a$ \\
$b$ & $b$ & $a$
\end{tabular}

Then, $\left(H,{ }_{14}\right)$ is an anticommutative semi-hypergroup, and the hyperoperation ${ }^{\circ}{ }_{14}$ is not associative, since $\left(a \circ{ }_{14} a\right) \circ{ }_{14} a=\{b\} \circ{ }_{14} a=\{b\} \neq a \circ{ }_{14}\left(a \circ{ }_{14} a\right)=a \circ{ }_{14}\{b\}=\{a\}$.

(AC) is valid, since $a \circ{ }_{14} b=\{a\} \neq b \circ{ }_{14} a=\{b\}$.

Definition 15. An anticommutative hypergroup is a hypergroup that satisfies (AR).

\section{Example 10}

(i) Let $H=\{a, b\}$. Define the hyperoperation ${ }^{\circ}{ }_{15}$ on $H$ with Cayley's table.

\begin{tabular}{l|ll}
$\circ_{15}$ & $a$ & $b$ \\
\hline$a$ & $H$ & $a$ \\
$b$ & $H$ & $H$
\end{tabular}

Then, $\left(H, \circ_{15}\right)$ is an anticommutative hypergroup. (AC) is valid, since $a \circ{ }_{15} b=\{a\} \neq b \circ{ }_{15} a=H$.

(ii) Let $H=\{a, b, c\}$. Define the hyperoperation ${ }^{\circ}{ }_{16}$ on $H$ with Cayley's table.

\begin{tabular}{c|ccc}
${ }_{16}$ & $a$ & $b$ & $c$ \\
\hline$a$ & $a$ & $a$ & $H$ \\
$b$ & $b$ & $b$ & $H$ \\
$c$ & $c$ & $c$ & $H$
\end{tabular}

Then, $\left(H,{ }_{16}\right)$ is an anticommutative hypergroup. The hyperoperation ${ }^{\circ}{ }_{16}$ is associative. Also, (AC) holds, since $a \circ{ }_{16} b=\{a\} \neq b \circ{ }_{16} a=\{b\}, \quad a \circ{ }_{16} c=$ $H \neq c \circ{ }_{16} a=\{c\}$, and $b \circ{ }_{16} c=H \neq c \circ{ }_{16} b=\{c\}$. (iii) Let $H=\{a, b, c\}$. Define the hyperoperation ${ }^{\circ}{ }_{17}$ on $H$ with Cayley's table.

\begin{tabular}{c|ccc}
$\circ_{17}$ & $a$ & $b$ & $c$ \\
\hline$a$ & $a$ & $b$ & $c$ \\
$b$ & $a$ & $b$ & $c$ \\
$c$ & $H$ & $H$ & $H$
\end{tabular}

Then, $\left(H,{ }^{\circ}{ }_{17}\right)$ is an anticommutative hypergroup, (AC) holds, since $a \circ{ }_{17} b=\{b\} \neq b \circ{ }_{17} a=\{a\}, \quad a \circ{ }_{17} c=\{c\} \neq$ $c \circ{ }_{17} a=H$, and $b \circ{ }_{17} c=\{c\} \neq c \circ{ }_{17} b=H$.

Note that every anti-semihypergroup, antihypergroup, anticommutative semi-hypergroup, and anticommutative hypergroup are anti-hyperalgebraic systems.

In the following results, we use hyperoperation instead of neutro-hyperoperation.

Note that if $(H, \circ)$ is a neutro-semihypergroup and $(G, \circ)$ is an anti-semihypergroup, then $(H \cap G, \circ)$ is not a neutro-semihypergroup, but it is an anti-semihypergroup. Also, let $\left(H,{ }^{\circ} H\right)$ be a neutro-semihypergroup, $\left(G, \circ_{G}\right)$ be an anti-semihypergroup, and $H \cap G=\varnothing$. Define hyperoperation $\circ$ on $H \uplus G$ by

$$
x \circ y=\left\{\begin{array}{cc}
x \circ_{H} y, & \text { if } x, y \in H, \\
x \circ_{G} y, & \text { if } x, y \in G, \\
\{x, y\}, & \text { otherwise. }
\end{array}\right\} .
$$

Then, $(H \uplus G, \circ)$ is a neutro-semihypergroup, but it is not an anti-semihypergroup.

Proposition 1. Let $(H, \circ)$ be an antisemihypergroup and $e \in H$. Then, $(H \cup\{e\}, *)$ is a neutrosemihypergroup, where * is defined on $H \cup\{e\}$ by

$$
x * y=\left\{\begin{array}{ll}
x \circ_{H} y, & \text { if } x, y \in H, \\
\{e, x, y\}, & \text { otherwise. }
\end{array}\right\} .
$$

Proof. It is straightforward.

Proposition 2. Let $(H, \circ)$ be a commutative hypergroupoid. Then, $(H, \circ)$ cannot be an anti-semihypergroup.

Proof. Let $a \in H$. Then, $a \circ(a \circ a)=(a \circ a) \circ a$, so $(H, \circ)$ cannot be an anti-semihypergroup.

Corollary 1. Let $(H, \circ)$ be a hypergroupoid, and there exists $a \in H$ such that $a^{\circ}$ a commuted with $a$. Then, $(H, \circ)$ cannot be an anti-semihypergroup.

Corollary 2. Let $(H, \circ)$ be a hypergroupoid with a scalar idempotent, i.e., there exists $a \in H$ such that $a^{\circ} a=a$. Then, $(H, \circ)$ cannot be an anti-semihypergroup.

Proposition 3. Let $\left(H, \circ_{H}\right)$ and $\left(G, \circ_{G}\right)$ be two neutrosemihypergroups (resp. anti-semihypergroups). Then, $(H \times$ 
$G, *$ ) is a neutro-semihypergroup (resp. anti-semihypergroups), where $*$ is defined on $H \times G$. For any $\left(x_{1}, y_{1}\right),\left(x_{2}, y_{2}\right) \in H \times G$,

$$
\left(x_{1}, y_{1}\right) *\left(x_{2}, y_{2}\right)=\left(x_{1}{ }^{\circ} x_{2}, y_{1}{ }_{G} y_{2}\right) \text {. }
$$

Note that if $(H, \circ)$ is a neutro-semihypergroup, then if there is a nonempty set $H_{1} \subseteq H$, such that $\left(H_{1}, \circ\right)$ is a semihypergroup, we call it Smarandache semi-hypergroup.

Suppose $\left(H,{ }^{\circ} H\right)$ and $\left(G,{ }^{\circ}\right)$ are two hypergroupoids. A function $f: H \longrightarrow G$ is called a homomorphism if, for all $a, b \in H, f\left(a \circ{ }_{H} b\right)=f(a){ }_{G} f(b)$ (see $[21,22]$, for details).

Proposition 4. Let $\left(H,{ }^{\circ} H\right)$ be a semi-hypergroup, $\left(G,{ }^{\circ}{ }_{G}\right)$ be a neutro-hypergroup, and $f: H \longrightarrow G$ be a homomorphism. Then, $\left(f(H),{ }^{\circ}\right)$ is a semi-hypergroup, where $f(H)=\{f(h): h \in H\}$.

Proof. Assume that $\left(H,{ }^{\circ}{ }_{H}\right)$ is a semi-hypergroup and $x, y, z \in f(H)$. Then, there exist $h_{1}, h_{2}, h_{3} \in f(H)$ such that $f\left(h_{1}\right)=x, f\left(h_{2}\right)=y$, and $f\left(h_{3}\right)=z$, so we have

$$
\begin{aligned}
x{ }^{\circ}\left(y \circ{ }_{G} z\right) & =f\left(h_{1}\right) \circ{ }_{G}\left(f\left(h_{2}\right){ }^{\circ}\left(h_{3}\right)\right) \\
& =f\left(h_{1}\right) \circ{ }_{G} f\left(h_{2} \circ{ }_{H} h_{3}\right)=f\left(h_{1} \circ{ }_{H}\left(h_{2} \circ{ }_{H} h_{3}\right)\right) \\
& =f\left(\left(h_{1} \circ{ }_{H} h_{2}\right) \circ{ }_{H} h_{3}\right)=f\left(h_{1} \circ{ }_{H} h_{2}\right){ }_{G} f\left(h_{3}\right) \\
& =\left(f\left(h_{1}\right) \circ{ }_{G} f\left(h_{2}\right)\right) \circ{ }_{G} f\left(h_{3}\right)=\left(x \circ{ }_{G} y\right) \circ{ }_{G} z .
\end{aligned}
$$

Then, $\left(f(H),{ }^{\circ}{ }_{G}\right)$ is a semi-hypergroup.

Definition 16. Let $\left(H,{ }^{\circ} H\right)$ and $\left(G,{ }^{\circ}{ }_{G}\right)$ be two hypergroupoids. A bijection $f: H \longrightarrow G$ is an isomorphism if it conserves the multiplication (i.e., $\left.f\left(a \circ{ }_{H} b\right)=f(a){ }_{\circ}{ }_{G} f(b)\right)$ and write $H \cong G$. A bijection $f: H \longrightarrow G$ is an antiisomorphism if for all $a, b \in H, f\left(a \circ{ }_{H} b\right) \neq f(b) \circ_{G} f(a)$. A bijection $f: H \longrightarrow G$ is a neutroisomorphism if there exist $a, b \in H, f\left(a \circ{ }_{H} b\right)=f(b){ }^{\circ}{ }_{G} f(a)$, i.e., degree of truth $(T)$, there exist $c, d \in H$ and $f\left(c{ }^{\circ}{ }_{H} d\right)$ or $f(c){ }^{\circ}{ }_{G} f(d)$ are indeterminate, i.e., degree of indeterminacy $(I)$, and there exist $e, h \in H, \quad f\left(e \circ{ }_{H} h\right) \neq f(e){ }_{G} f(h)$, i.e., degree of falsehood $(F)$, where $(T, I, F)$ are different from $(1,0,0)$ and $(0,0,1)$, and $T, I, F \in[0,1]$.

Let $\circ$ be a hyperoperation on $H=\{a, b\}$ and ( $\left.A_{11}, A_{12}, A_{21}, A_{22}\right)$ inside of Cayley's table.

$$
\begin{array}{c|cc}
\circ & a & b \\
\hline a & \mathrm{~A}_{11} & \mathrm{~A}_{12} \\
b & \mathrm{~A}_{21} & \mathrm{~A}_{22}
\end{array}
$$

Lemma 1 (see [5]). Let $\left(H=\{a, b\},{ }^{\circ}\right)$ and $\left(G=\left\{a^{\prime}, b^{\prime}\right\},{ }_{G}\right)$ be hypergroupoids with Cayley's tables $(A, B, C, D)$ and $\left(A^{\prime}, B^{\prime}, C^{\prime}, D^{\prime}\right)$, respectively. Then, $H \cong G$ if and only if, for all $i, j \in\{1,2\}, A_{i j}=A_{i j}^{\prime}$ or

$$
A_{i j}^{\prime}=\left\{\begin{array}{ll}
A_{i j}^{d}, & \text { if } A_{i j}=H, \\
G \backslash A_{i j}^{\prime}, & \text { if } A_{i j} \neq H,
\end{array}\right\},
$$

where $A_{11}^{d}=A_{22}, A_{12}^{d}=A_{12}, A_{21}^{d}=A_{21}$, and $A_{22}^{d}=A_{11}$.

Lemma 2 (see [6]). If $(H, \circ)$ is a hypergroupoid, then $(H, *)$ is a hypergroupoid when $x * y=y \circ x$ for all $x, y \in H$.

$(H, *)$ in Lemma 2 is called dual hypergroupoid of $(H, \circ)$.

Theorem 1. Let $(H=\{a, b\}, \circ)$. Then, $(H, \circ) \cong(H, *)$ if and only if $(H, \circ)$ is anticommutative.

Lemma 3. There exist 4 anticommutative anti-semihypergroup of order 2 (up to isomorphism).

Proof. Let $(H, \circ)$ be an anticommutative antisemihypergroup. By Corollary 2, we have $a \circ a \neq a$ and $b \circ b \neq b$. Also, $a \circ b \neq b \circ a$. Consider the following.

If $a \circ a=H$, then $a \circ(a \circ a)=a \circ H=H=H \circ a=$ $(a \circ a) \circ a$, a contradiction. Then, we get $a \circ a=b$ and $b \circ b=a$.

Now, we have

Case 1. If $a \circ b=a$, then $b \circ a=H$ or $b \circ a=b$, so we get $(b, a, b, a)$ and $(b, a, H, a)$ are two antisemihypergroups

Case 2. If $a \circ b=b$, then $b \circ a=H$ or $b \circ a=a$, so we get $(b, b, a, a)$ and $(b, b, H, a)$ are two antisemihypergroups

Case 3. If $a \circ b=H$, then $b \circ a=a$ or $b \circ a=b$, so we get $(b, H, a, a)$ and $(b, H, b, a)$ are two antisemihypergroups

It can be see that $(b, a, H, a) \cong(b, H, b, a)$ and $(b, H, a, a) \cong(b, b, H, a)$. Therefore, $(b, b, a, a), \quad(b, a, b, a)$, $(b, a, H, a)$, and $(b, H, a, a)$ are 4 nonisomorphic antisemihypergroups of order 2 .

Corollary 3. There exists two nonisomorphic antisemigroups of order 2: $(b, b, a, a)$ and $(b, a, b, a)$. Antisemigroup $(b, b, a, a)$ is the dual form of the anti-semigroup $(b, a, b, a)$.

Corollary 4. There exists two nonisomorphic antisemihypergroups of order 2: $(b, a, H, a)$ and $(b, H, a, a)$. Anti-semihypergroup $(b, a, H, a)$ is the dual form of the antisemihypergroup $(b, H, a, a)$.

Theorem 2. Let $(H, \circ)$ be a hypergroupoid of order 2. Then, $(H, \circ)$ does not have (NR) or (AR).

Proof. Let $H=\{a, b\}$. Suppose $H a \neq H, a H \neq H$, and $H a \neq a H$. Hence, $H a=\{a\}$ or $H a=\{b\}$. First, give $H a=\{a\}$, then $a H \neq H$ and $H a \neq a H$ implies that $a H=\{b\}$. Then, $a \circ a \subseteq H a=\{b\}$ and $a \circ a \subseteq H a=\{a\}$. Therefore, $\{b\}=a \circ a \circ a$ $=\{a\}$, and this is a contradiction. In the similar way, we obtain $H b \neq H, b H \neq H$, and $H b \neq b H$, a contradiction.

Using Lemmas 1 and 2 and Theorem 1, we can find 45 nonisomorphic classes hypergroupoids of the order 2 . We characterize these 45 classes in Table 1. 
Note that semi-hypergroups, hypergroups, and fuzzy hypergroups of order 2 are enumerated in $[7,11,12]$.

We obtain anti-semihypergroups and neutrosemihypergroups of order 2 and the classification of the hypergroupoids of order 2 (classes up to isomorphism).

R, NR, AR, A, NA, AA, C, NC, and AC in Table 1 are denoted in Sections 2 and 3.

A result from Table 1 confirms the enumeration of the hyperstructure of order $2[11,23,24]$, which is summarized as follows.

\section{Conclusion and Future Work}

In this paper, we have studied several special types of hypergroups, neutro-semihypergroups, anti-semihypergroups, neutro-hypergroups, and anti-hypergroups. New results and examples on these new algebraic structures have been investigated. Also, we characterize all neutro-hypergroups and antihypergroups of order two up to isomorphism. These concepts can further be generalized.

Future research to be done related to this topic are

(a) Define neutro-quasihypergroup, anti-quasihypergroup, neutrocommutative quasi-hypergroup, and anticommutative quasi-hypergroup

(b) Define neutro-hypergroups, anti-hypergroups, neutrocommutative hypergroups, and anticommutative hypergroups

(c) Define and investigate neutroHv-groups, antiHvgroups, neutroHv-rings, and antiHv-rings

(d) It will be interesting to characterize infinite neutrohypergroups and anti-hypergroups up to isomorphism

(e) These results can be applied to other hyperalgebraic structures, such as hyper-rings, hyperspaces, hyper-BCK-algebra, hyper-BE-algebras, and hyper-K-algebras.

\section{Data Availability}

The data used to support the findings of this study are included within the article.

\section{Conflicts of Interest}

The authors declare that they have no conflicts of interest.

\section{References}

[1] F. Marty, Sur une Generalization de la Notion de Groupe, pp. 45-49, Huitieme Congress de Mathematiciens, Scandinaves, Stockholm, 1934.

[2] P. Corsini, Prolegomena of Hypergroup Theory, Aviani, Udine, Italy, 1993.

[3] M. Al-Tahan and B. Davvaz, "On Corsini hypergroups and their productional hypergroups," The Korean Journal of Mathematics, vol. 27, no. 1, pp. 63-80, 2019.

[4] P. Corsini and V. Leoreanu-Fotea, Applications of Hyperstruture Theory, Springer Science+Business Media, Berlin, Germany, 2003.
[5] B. Davvaz and V. Leoreanu-Fotea, Hyperring Theory and Applications, International Academic Press, Cambridge, MA, USA, 2007.

[6] B. Davvaz and T. Vougiouklis, A Walk through Weak Hyperstructures; $H_{v}$-Structure, World Scientific Publishing, Hackensack, NJ, USA, 2019.

[7] T. Vougiouklis, Cyclic Hypergroups, Ph. D Thesis, Democritous University of Thrace, Komotini, Greece, 1980.

[8] B. Davvaz, Semihypergroup Theory, Elsevier, Amsterdam, Netherlands, 2016.

[9] B. Davvaz, Polygroup Theory and Related Systems, World Scientific, Singapore, 2013.

[10] B. Davvaz and I. Cristea, Fuzzy Algebraic Hyperstructures; An Introduction, Springer, Berlin, Germany, 2015.

[11] C. G. Massouros and G. G. Massouros, "On 2-element fuzzy and mimic fuzzy hypergroups," AIP Conference Proceedings, vol. 1479, pp. 2213-2216, 2012.

[12] T. Vougiouklis, Hyperstructures and Their Representations, Hadronic Press, Palm Harbor, FL, USA, 1994.

[13] S. Hoskova-Mayerova, M. Al Tahan, and B. Davvaz, "Fuzzy multi-hypergroups," Mathematics, vol. 8, no. 2, p. 244, 2020.

[14] F. Smarandache, "Neutroalgebra is a generalization of partial algebra," International Journal of Neutrosophic Science, vol. 2, no. 1, pp. 8-17, 2020.

[15] F. Smarandache, "Introduction to neutroalgebraic structures and antialgebraic structures," in Advances of Standard and Nonstandard Neutrosophic Theories, Pons Publishing House, Brussels, Belgium, 2019.

[16] F. Smarandache, "Introduction to NeutroAlgebraic structures and AntiAlgebraic structures (revisited)," Neutrosophic Sets and Systems, vol. 31, pp. 1-16, 2020.

[17] F. Smarandache, "Extension of hypergraph to nsuperhypergraph and to plithogenic n-superhypergraph, and extension of hyperalgebra to n-ary (classical-/neutro-/anti-) hyperalgebra," Neutrosophic Sets and Systems, vol. 33, pp. 290296, 2020

[18] A. A. A. Agboola, "Introduction to NeutroGroups," International Journal of Neutrosophic Science, vol. 6, no. 1, pp. 41-47, 2020.

[19] M. Al-Tahan, F. Smarandache, and B. Davvaz, "Neutro ordered algebra: applications to semigroups," Neutrosophic Sets and Systems, vol. 39, pp. 133-147, 2021.

[20] F. Smarandache, Neutrosophy/Neutrosophic Probability, Set, and Logic, American Research Press, Santa Fe, NM, USA, 1998.

[21] R. Ameri and M. M. Zahedi, "Hyperalgebraic system," Italian Journal of Pure and Applied Mathematics, vol. 6, pp. 21-32, 1999.

[22] P. Corsini and V. Leoreanu-Fotea, "Hypergroups and binary relations," Algebra Universalis, vol. 43, pp. 321-330, 2000.

[23] R. Bayon and N. Lygeros, "Catégories spécifiques dhypergroupes dordre 3, in: structure elements of hyper-structures," pp. 17-33, Spanidis, Xanthi, Greece, 2005.

[24] S. Worawiset, J. Koppitz, and S. Chotchaisthit, "The class of all semigroups related to semihypergroups of order 2," Mathematica Slovaca, vol. 69, no. 2, pp. 371-380, 2019. 